\title{
Reservoir Computing Based on VCSEL Subject to Optical Injection and Arbitrary Polarization Optical Feedback
}

\author{
Yushuang HOU, ${ }^{\mathrm{a}, \mathrm{b}}$, Wenyu SUI ${ }^{\mathrm{b}}$ and Dianzuo YUE ${ }^{\mathrm{a}, 1}$ \\ ${ }^{a}$ School of Mathematics and Information Science \& Technology, Hebei Normal \\ University of Science and Technology, China \\ ${ }^{\mathrm{b}}$ School of Information Engineering, Inner Mongolia University of Science and \\ Technology, China
}

\begin{abstract}
We introduce a new reservoir computer based on vertical cavity surface emitting semiconductor laser (VCSEL) with optical injection and arbitrary polarization optical feedback. By changing the feedback optical polarization angle and under parallel or orthogonal optical injection, we demonstrate that the introduction of new parameter (polarization angle) increases one degree of freedom of the system, making the parameters adjustment space much wider than parallel or orthogonal optical feedback ones. Via Nonlinear channel equalization benchmark task, the performance of such RC system is investigated, and the results indicate the system can yield good performance that can be compared with or better than similar reservoirs.
\end{abstract}

Keywords. Reservoir computing (RC), nonlinear channel equalization, vertical cavity surface emitting semiconductor laser (VCSEL)

\section{Introduction}

Reservoir computing (RC) as a new method inspired by neural networks has received extensive attention in recent years. It originated from recurrent neural networks, but has the advantage of easy training. Generally, a traditional RC comprises of three layers, named as input layer, reservoir and output layer. The connection of the input layer and the reservoir layer is randomly selected, and then fixed, only the readout layer need to be trained. Notably, based on a single nonlinear component under delayed feedback, a novel RC system named as time delayed RC is proposed in 2011 [1]. Such time delayed RC system possesses the superiority of easy hardware-implementation. Semiconductor lasers (SLs) with time delayed feedback are very promising for high-speed implementation of RC and high dimensional transformation of input signal.

Compared with edge emitting lasers, vertical cavity surface emitting semiconductor lasers (VCSELs) have some unique advantages due to their different structures, which makes VCSELs as reservoirs easier to realize some basic properties that RC must have. Recently, much research work has been reported on the rich nonlinear characteristics exhibited by VCSELs under conditions of optical injection and optical feedback, and the

${ }^{1}$ Corresponding Author, Dianzuo YUE, School of Mathematics and Information Science \& Technology, Hebei Normal University of Science and Technology, China; Email: 2095344322@qq.com. 
switching between two polarization states, their nonlinear characteristics are one of the necessary conditions to constitute a reservoir computer. In this study, we propose a new method to constructing reservoir computer using VCSEL subject to optical injection and arbitrary polarization optical feedback. Via Nonlinear channel equalization task [2], the performance of such RC system is investigated, and the influences of some typical parameters on the performance have been analyzed.

\section{System Model}

Figure 1(a) is the schematic diagram of our proposed RC system. The main laser is a tunable laser, we consider that the wavelength of the main laser is adjusted to be the same as the VCSEL (1550nm), so here is no frequency detuning between the injected optical and the VCSEL. Polarization controller (PC) can adjust the polarization direction of the injected light. We consider two ways of injection which are defined as parallel and orthogonal injection, which means the injection polarization direction consistent with the $\mathrm{X}$ polarization (XP) or Y polarization (YP) mode of the VCSEL. The two attenuators can control the strength of inject and feedback light respectively to get the best working condition. In the feedback loop of VCSEL, we use a tunable polarization wave plate (TPW) to adjust the polarization angle of feedback light. The Mach-Zehnder (MZ) intensity modulator and arbitrary waveform generator (AWG) can input the signal and mask. $\theta_{p}=0^{\circ}$ represents only XP mode can pass through TPW and no YP element. $\theta_{p}=$ $90^{\circ}$ represents only YP mode can pass through TPW and no XP element. Therefore, when $\theta_{p}$ changes from $0^{\circ}$ to $90^{\circ}$ as shown in figure $1(\mathrm{~b})$, the output light of VCSEL is only the components $E_{x} \cos \left(\theta_{p}\right)$ of XP mode and $E_{y} \sin \left(\theta_{p}\right)$ of YP mode can pass through TPW, then it can be expressed as $E_{f}=E_{x} \cos \left(\theta_{p}\right)+E_{y} \sin \left(\theta_{p}\right)$. When this light is feedback into VCSEL, the feedback electric field amplitudes are given by:

$$
\begin{aligned}
& E_{f}^{X}(t-\tau)=E_{f}(t-\tau) \cos \left(\theta_{p}\right) \\
& E_{f}^{Y}(t-\tau)=E_{f}(t-\tau) \sin \left(\theta_{p}\right)
\end{aligned}
$$

(a)

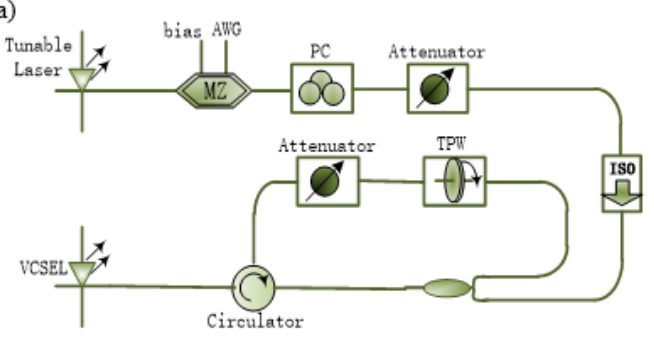

(b)

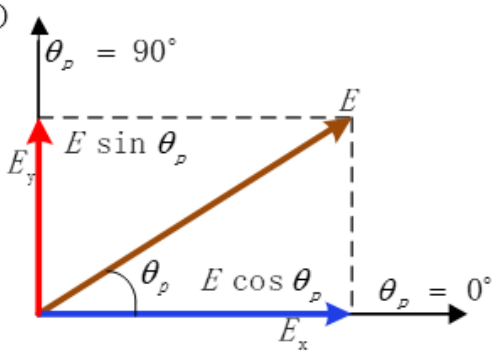

Figure 1. (a)Schematic diagram of RC based on VCSEL subject to optical injection and arbitrary polarization optical feedback. (b) Schematic diagram of the relationship between two polarization modes of VCSEL under feedback of variable polarization angle. $E_{\mathrm{x}}$ and $E_{\mathrm{y}}$ are slow varying electric field amplitude of XP mode and YP mode. 
The simulation model we used is based on the spin-flip model (SFM), which descripts slowly varying complex electric field amplitudes and the carrier number in the laser cavity [3-4]. It is extended with optical injection term and arbitrary polarization optical feedback term [5]. The rate equations of the extended model are given by:

$$
\begin{aligned}
& \frac{d E_{\mathrm{x}}}{d t}=k(1+i a)\left[(N-1) E_{x}+i n E_{y}\right]-\left(\gamma_{a}+i \gamma_{p}\right) E_{x}+k_{x} k_{i n j} E_{i n j}(t) e^{\left(\omega_{i n j}-\omega_{0}\right) t} \\
& +\eta E_{x}(t-\tau) \cos ^{2}\left(\theta_{p}\right) e^{-i \omega \tau}+\eta E_{y}(t-\tau) \cos \left(\theta_{p}\right) \sin \left(\theta_{p}\right) e^{-i \omega \tau}+F_{\mathrm{x}} \\
& \frac{d E_{\mathrm{y}}}{d t}=k(1+i a)\left[(N-1) E_{y}+i n E_{x}\right]-\left(\gamma_{a}+i \gamma_{p}\right) E_{y}+k_{y} k_{i n j} E_{i n j}(t) e^{\left(\omega_{i n j}-\omega_{0}\right) t} \\
& +\eta E_{y}(t-\tau) \sin ^{2}\left(\theta_{p}\right) e^{-i \omega \tau}+\eta E_{x}(t-\tau) \sin \left(\theta_{p}\right) \cos \left(\theta_{p}\right) e^{-i \omega \tau}+F_{y} \\
& \frac{d N}{d t}=\gamma_{N}\left[\mu-N\left(1+\left|E_{x}\right|^{2}+\left|E_{y}\right|^{2}\right)+i n\left(E_{x} E_{y}^{*}-E_{y} E_{x}^{*}\right)\right] \\
& \frac{d n}{d t}=-\gamma_{s} n-\gamma_{N}\left[n\left(\left|E_{x}\right|^{2}+\left|E_{y}\right|^{2}\right)+i N\left(E_{y} E_{x}^{*}-E_{x} E_{y}^{*}\right)\right]
\end{aligned}
$$

where the subscript $\mathrm{x}$, $\mathrm{y}$ represents two polarization modes of XP and YP, and $N$ denotes the number of carriers in the laser cavity. $\omega_{i n j}$ is the injected optical angular frequency, $\omega_{0}$ is the angular frequency of VCSEL, and $k$ is the decay rate of the electric field. $\gamma_{p}$ is the linear birefringence rate and $\gamma_{a}$ is the rate of linear dichroism. $\alpha$ is linewidth enhancement factor, $\gamma_{N}$ is the decay rate of the carrier population, $\gamma_{s}$ is the spin-flip relaxation rate. Here we will consider two ways of injection, parallel injection and orthogonality injection. The injected optical polarization direction is consistent with XP mode $\left(k_{x}=1, k_{y}=0\right)$ and YP mode $\left(k_{x}=0, k_{y}=1\right)$ respectively. $E_{i n j}$ is electric field amplitude of the injected optical, $k_{i n j}$ is injection coefficient and we can change it from $0 \mathrm{~ns}^{-1}$ to 300 $\mathrm{ns}^{-1}$. And the input signal modulated by $\mathrm{MZ}$ can be expressed as $E_{i n j}=P_{i n j}^{1 / 2}\left(1+\mathrm{e}^{i V / V} \pi\right), V$ is the voltage of signal which output by AWG, and between $\left[-\pi V_{\pi} ; \pi V_{\pi}\right], V_{\pi}$ is the half wave voltage of MZ. In our simulation, we set $P_{i n j}=1, V_{\pi}=1 . F_{x}$ and $F_{y}$ which are the last item in equation (3) and (4) represent spontaneous emission noise, and can be expressed mathematically as:

$$
\begin{aligned}
& F_{x}=\sqrt{\frac{\beta_{s p}}{2}}\left(\sqrt{N+n} \xi_{1}+\sqrt{N-n} \xi_{2}\right) \\
& F_{y}=-i \sqrt{\frac{\beta_{s p}}{2}}\left(\sqrt{N+n} \xi_{1}-\sqrt{N-n} \xi_{2}\right)
\end{aligned}
$$

here $\xi_{1}$ and $\xi_{2}$ are Gaussian white noise, $\beta_{s p}$ is the spontaneous emission factor. In our simulation $\alpha=3, k=300 \times 10^{9} \mathrm{~s}^{-1}, \gamma_{\mathrm{a}}=1 \times 10^{9} \mathrm{~s}^{-1}, \gamma_{\mathrm{p}}=192.1 \times 10^{9} \mathrm{~s}^{-1}, \gamma_{\mathrm{N}}=1 \times 10^{9} \mathrm{~s}^{-1}, \lambda=1550 \times 10^{-}$ ${ }^{9} \mathrm{~m}, \mathrm{c}=2.998 \times 10^{8} \mathrm{~m} \cdot \mathrm{s}^{-1}, \beta_{\mathrm{sp}}=4.5 \times 10^{-4} \mathrm{~ns}^{-1}$. We use the method of fourth order Runge-Kutta to solve differential equations, and the integration step set to $2 \mathrm{ps}$. 
In the reservoir, the delay time of the feedback loop is defined as $\tau$, and then $\theta$ is defined as time interval between virtual nodes which evenly distributed along the feedback loop. So, the number of nodes $N=\tau / \theta$. we set VCSEL work current $\mu=1.3 I_{t h}$.

\section{Results and Discussion}

We use 3000 points to train the readout weights, and 6000 points for the test. We consider two kinds of injection of parallel mode and orthogonality mode. First, we inject the light as parallel mode, fix the polarization angle $\theta_{p}=0, \theta=0.02 \mathrm{~ns}, N=40$. In the meanwhile, the SNR is set to $20 \mathrm{~dB}$, and then we explore the optimal combination range of feedback strength and injection coefficient.
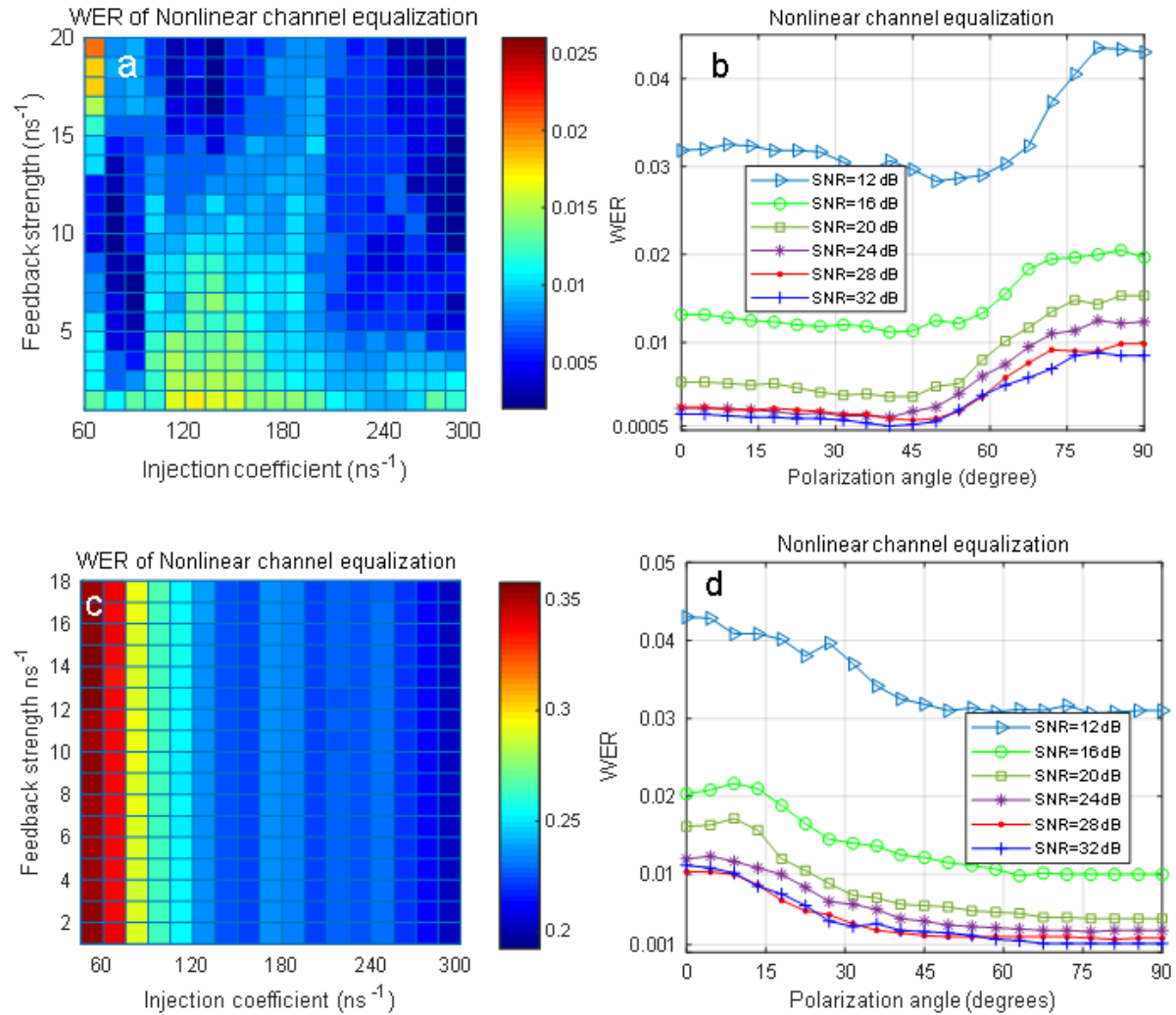

Figure 2. Nonlinear channel equalization testing result, with parameters $\theta=0.02 \mathrm{~ns}$ and number of nodes $N=40$, SER as a function of (a) feedback strength $\eta$ and injection coefficient $k_{i n j}$ under parallel injection mode, $\mathrm{SNR}=20 \mathrm{~dB}, \theta_{p}=0$, (b) polarization angle $\theta_{p}$ under parallel injection, $\eta=11 \mathrm{~ns}^{-1}$ and $k_{i n j}=90 \mathrm{~ns}^{-1}$, under six kinds of SNR, (c) feedback strength $\eta$ and injection coefficient $k_{i n j}$ under orthogonality injection mode, SNR $=20 \mathrm{~dB}$, $\theta_{p}=45^{\circ}$, (d) polarization angle $\theta_{p}$ under orthogonality injection, $\eta=10 \mathrm{~ns}^{-1}$ and $k_{i n j}=165 \mathrm{~ns}^{-1}$, under six kinds of SNR.

As shown in figure 2(a), we find three areas that achieved good performance, i.e. $k_{\text {inj }}$ between $60 \mathrm{~ns}^{-1}$ to $90 \mathrm{~ns}^{-1}$ with $\eta$ around $10 \mathrm{~ns}^{-1}, k_{\text {inj }}$ near $150 \mathrm{~ns}^{-1}$ with $\eta$ above $15 \mathrm{~ns}^{-1}$ and $k_{\text {inj }}$ above $270 \mathrm{~ns}^{-1}$ with $\eta$ above $15 \mathrm{~ns}^{-1}$. Then we select $k_{\text {inj }}=90 \mathrm{~ns}^{-1}$ and $\eta=11 \mathrm{~ns}^{-1}$. We test the performance of reservoir as we change $\theta_{p}$ from $0^{\circ}$ to $90^{\circ}$ under different SNR. The result is shown in figure 2(b). In this figure, we can see that in several kinds of SNR this 
reservoir can achieve good performance, and when $\theta_{p}$ is below $45^{\circ}$, the SER is much lower than $\theta_{p}>45^{\circ}$, and the best performance is achieved around $\theta_{p}=45^{\circ}$. With $\mathrm{SNR}=32 \mathrm{~dB}$, the best $\mathrm{SER}$ is about $5 \times 10^{-4}$.

Next, we change the injection mode as orthogonality mode, and also set $\theta=0.02 \mathrm{~ns}$, $N=40$, and fixed $\mathrm{SNR}=20 \mathrm{~dB}$. Set polarization angle $\theta_{p}=45^{\circ}$. As we change the injection coefficient $k_{\text {inj }}$ and feedback strength $\eta$ we can identify the trend of SER.

As shown in figure 2(c), in this situation, the effect of feedback strength is not obvious, and the injection coefficient dominated the SER trend. In the orthogonality injection mode, we can easily find out the most suitable parameters. The best performance can achieve with $k_{i n j}$ near $150 \mathrm{~ns}^{-1}$ and $300 \mathrm{~ns}^{-1}$. We then fixed $k_{i n j}=165 \mathrm{~ns}^{-1}$ and $\eta=10 \mathrm{~ns}^{-1}$ and run the reservoir in different value of SNR and polarization angle. It can be shown in figure 2(d). We can also achieve desired output but the best range is located near $\theta_{p}=90^{\circ}$, and when $\mathrm{SNR}=32 \mathrm{~dB}$, the minimum SER is $1 \times 10^{-3}$. We can see that in the test of Nonlinear channel equalization, when SNR is relatively large ( $\mathrm{SNR}=12 \mathrm{~dB})$, the SER still below 0.05. In fact, we have also tested the extreme noise environment $(\mathrm{SNR}=0 \mathrm{~dB})$, and achieved SER is below 0.5 . Note that we set VCSEL spontaneous emission rate to realistic value $\beta_{s p}=4.5 \times 10^{-4} \mathrm{~ns}^{-1}$ throughout the test, so we can see this reservoir shows strong anti-interference ability.

\section{Conclusion}

In conclusion, the new implementation of reservoir using 1550-nm VCSEL with optical injection and under arbitrary polarization angle optical feedback is proposed and investigated in this article. We discussed the parallel and orthogonality injection mode of VCSEL respectively, and with the optical feedback of arbitrary polarization by adjusting the angle of the adjustable polarization plate. Nonlinear channel equalization task is utilized to test the system, and the simulation results show that the system exhibits excellent anti-jamming performance. In the two injection modes, when the injection coefficient and the feedback strength are determined, the better effect can be obtained by adjusting the polarization angle in the region of $0^{\circ}$ to $90^{\circ}$. We also note that when XP mode dominated mode and YP mode is suppressed, adjusting the injected light parallel with the direction of YP mode, the reservoir can achieve good performance in a wide range of parameters, which provides a useful basis for experiment implementation. Furthermore, compared with the result obtained in similar time delayed RC system with single optical feedback, this system possesses better prediction performance.

\section{Acknowledgements}

This work is supported by the National Natural Science Foundation of China (NSFC) under grant no. 62065015, and in part by the Natural Science Foundation of Inner Mongolia Autonomous Region of China under Grant 2019MS06022 and 2021LHMS01006. 


\section{References}

[1] Appeltant L, Soriano MC, Van der Sande G, Danckaert J, Massar S, Dambre J, Schrauwen B, Mirasso $\mathrm{CR}$, Fischer I. Information processing using a single dynamical node as complex system. Nature Communication. 2011 Sep; 2: 468.

[2] Duport F, Schneider B, Smerieri A, Haelterman M, Massar S. All-optical reservoir computing. Optics Express. 2012 Sep; 20(20): 22783-95.

[3] Pan ZG, Jiang SJ, Dagenais M, Morgan RA, Kojima K, Asom MT, Leibenguth RE. Optical injection induced polarisation bistability in vertical-cavity surface-emitting lasers. Applied Physics Letters. 1993 May; 63(22): 2999-3001.

[4] Gatare I, Sciamanna M, Buesa J, Thienpont H, Panajotov K. Nonlinear dynamics accompanying polarization switching in vertical-cavity surface-emitting lasers with orthogonal optical injection. Applied Physics Letters. 2006 Mar; 88(10): 101106-1-1-3.

[5] Al-Seyab R, Schires K, Hurtado A, Henning ID, Adams MJ. Dynamics of VCSELs Subject to Optical Injection of arbitrary polarization. IEEE Journal of Selected Topics in Quantum Electronics. 2013 Jul; 19(4): 1700512. 